\title{
Masquelet Technique: Effects of Topical Mechanical Stability on the Formation of Masquelet Membrane in a Rabbit Radial Defect Model
}

Xie Jie

xiangya hospital central south university

Liu Donghao

Xiangya Hospital Central South University

Wang Haoyi

Xiangya Hospital Central South University

Long Haitao

xiangya hospital central south university

Zhu Yong

xiangya hospital central south university

Hu Yihe

xiangya hospital central south university

Cheng Liang

xiangya hospital central south university

Min Zeng ( $\sim$ xy_zengmin@163.com)

Xiangya Hospital Central South University

\section{Research article}

Keywords: Masquelet Technique, Induced membrane, PMMA, Mechanical stability, Osteogenesis, Vascularization

Posted Date: January 2nd, 2020

DOl: https://doi.org/10.21203/rs.2.19835/v1

License: (c) (i) This work is licensed under a Creative Commons Attribution 4.0 International License.

Read Full License 


\section{Abstract}

Purpose The Masquelet technique is a commonly treatment strategy for segmental bone defects. Still, the exact generating mechanism is unknown. This study intends to explore the effects of topical mechanical stability on the formation of Masquelet membrane.

Methods Thirty-six New Zealand white rabbits were evenly randomized into three groups. Segmental left radius shaft defect (length, $9 \mathrm{~mm}$ ) was created in all rabbits. Bone defects were filled with polymethylmethacrylate (PMMA) in Non-fixation group, and with PMMA fixed with plates in Fixation group, and subjected to no disposal in control group. The stability of PMMA and plates were monitored via X-ray. And the membranes were excised for further Histological, IHC (Ki67, COL I, and CD31), and Western-Blotting analysis (RUNX2, ALP, VEGFA, and TGF- $\beta 1$ ), 4 and 6 weeks post-operatively

Results X-ray revealed no sign of plates loosening, or shift of PMMA. Pathological examinations suggested that vascularized and osteogenic membranes were formed around PMMA. IHC and WesternBlotting analysis revealed that both Fixation and Non-fixation group exerted significant effects on the expression of Ki67, COL I, and CD31 positive cells, as well as the protein expression of osteogenic (RUNX2, ALP) and angiogenic (VEGFA, TGF- $\beta 1$ ) factors. And compared with membrane in Non-fixation group, Fixing PMMA spacer with plates caused a significant increase in osteogenic and angiogenic expression, 4 and 6 weeks post-operatively.

Conclusion The present study indicates that rigid fixation provided by internal plate in Masquelet technique positively alters the quality of membrane formed surrounding PMMA, in terms of significantly osteogenic and angiogenic potential.

\section{Introduction}

Segmental bone defects, caused by trauma, osteomyelitis, and tumor resection, has become a major health problem obfuscating orthopedist $(1-3)$. One strategy of growing concern in bone defect repairing is the Masquelet or induced membrane technique (4-6). Mixed results are shown in previous animal or human studies $(1,3,7,8)$. The key to bone repair relies on a vascularized and osteoblastic membrane formed during the first stage of Masquelet technique $(9,10)$. Still, the exact generating and regulatory mechanism of Masquelet membrane are unknown. In order to solve these problems, several studies show that the membrane has structural characteristics and osteogenic potentials. It is not just offering a physical barrier and a rich vascular network, but also numerous osteoprogenitor cells and a local source of key biochemical factors, such as BMP2, and TGF- $\beta(11-13)$. And previous researches have demonstrated that selecting a new model, such as spacer alternatives, micro-topography modification, local antibiotic usage, would affect the structure and function of membrane(13-18).

During the first phase of Masquelet, the bone defects filling with bone cement (polymethyl methacrylate, PMMA) spacer are fixed with internal plate, intramedullary nail, external fixator, casting, or nothing(12, 17, 19-21). But it is not sure that which fixation method is more appropriate for membrane formation in the 
first stage of Masquelet(13,22-24). It is known that the stiffness of fixation construct directly affects the jiggle of fracture fragments, subsequently impacts the healing of bone fracture. A relatively high level of rigid fixation results in intramembranous ossification, while less rigid fixation results in cartilaginous callus and endochonrdral ossification $(25,26)$. And there is no literature reported regarding whether there are differences in the membrane formation among various fixation pattern.

This study intends to explore the effects of topical mechanical stability on the formation of Masquelet membrane. Based on previous studies, we hypothesize that an increase in fixation strength will results in superior Masquelet membrane with significance in osteogenic and angiogenic factors expression.

\section{Materials And Methods}

\section{Animal models}

All procedures were done with the approval of our Medical Ethics Committee. Thirty-six New Zealand white rabbits, weighing approximately $2500-3000 \mathrm{~g}$ and aged 24-28 weeks, were evenly randomized into three groups (Fixation group, Non-fixation group, and control group). Segmental left radius shaft defect (length, $9 \mathrm{~mm}$ ) was created in all rabbits. And the bone defects were filled with PMMA (length, $9 \mathrm{~mm}$ ) only (Non-fixation group, $\mathrm{n}=12$ ), or PMMA fixed with plates (Synthes, USA) (Fixation group, $\mathrm{n}=$ 12), respectively. In the control group, the defects were subjected to no disposal (control group, $n=12$ ).

Xylazine hydrochloride was given to rabbits by intramuscular injection, and lidocaine was used for additional regional anesthesia before osteotomy. All rabbits were arranged on fixed frames, and the left forelimbs were shaved, prepared, and draped before surgery. After the skin, superficial fascia and muscles of the left forelimb were incised, exposing the shaft of radius. A $9 \mathrm{~mm}$ defect was created at approximately middle radius shaft with a high-speed power drill, and subsequently a cylindrical PMMA spacer ( $3 \mathrm{~mm} \varnothing \times 9 \mathrm{~mm})$ was placed in the defect in Fixation and Non-fixation group. A six-hole, $2.5 \mathrm{~mm}$ stainless steel plates (Synthes, USA) was applied to the radius shaft and secured in place with two proximal and two distal $1.5 \mathrm{~mm}$ cortical screws in Fixation group. Then tight suture of the fascia incision with 4- 0 absorbable sutures ensures stability of PMMA. The skin wounds were closed with interrupted 4 - 0 Mersilk sutures. Cefotaxime Sodium 20,000 IU/kg was administered intramuscularly immediately, 24 and 48 hours post-operatively. (Fig. 1A-D)

Immediately, 4 and 6 weeks post-operatively, X-ray was used to evaluate the location of implants. Half of animals were sacrificed at 4 weeks after operation, and the other half were sacrificed at 6 weeks through air embolism. Through the former incision, the skin and muscles were dissected. Membranes formed around PMMA were excised completely. The membranes were divided into two parts, which were respectively fixed in $10 \%$ paraformaldehyde for later pathological examination and shock frozen $\left(-80^{\circ} \mathrm{C}\right)$ for later Western-Blotting analysis. (Fig. 1E-F)

\section{Radiography}


The stability of PMMA and plates (i.e. PMMA migration and plates loosening) were monitored via X-ray in all rabbits immediately, 4 and 6 weeks post-operatively.

\section{Histological and IHC analysis}

After embedding and sectioning, paraffin specimens were heated and subsequently removed by sequential washes (xylene 100\%, 20 min thrice; alcohol 100\%, 5 min: alcohol 95\%, 5 min; alcohol 85\%, $5 \mathrm{~min}$; alcohol 75\%, $5 \mathrm{~min}$; distilled water, $5 \mathrm{~min}$ ). Subsequently, the specimens were stained with haematoxylin/eosin ( $\mathrm{HE}$ ) and Alizarin red $\mathrm{S}$ to evaluate the histopathological characteristics.

Content of cell proliferation, osteogenesis, and vascularization in the induced membranes were measured using immunohistochemistry (IHC) analysis by detection of Ki67, COL I, and CD31 ( $\mathrm{n}=6 /$ group), respectively. Paraffin sections were first removed by sequential washes (xylene 100\%, 20 min thrice; alcohol 100\%, 5 min: alcohol 95\%, 5 min; alcohol 85\%, 5 min; alcohol 75\%, 5 min; distilled water, 5 min). Then, the processes of antigen retrieval, incubation of primary antibody (CD31, Ki67, and COL I), incubation of secondary antibody (anti-mouse, rabbit, mouse-IgG antibody-HRP polymer) were in progress. Finally, after DAB coloration, hematoxylin redyeing, and Alcohol dehydration, IHC sections were imaged under microscope (DMIL-LED, LEICA, Germany). An independent observer blinded to the group constituency analysed the images.

\section{Western blot analysis}

Osteogenic proteins (RUNX2 and ALP) and angiogenic proteins (VEGF and TGF- $\beta 1$ ) were measured using Western blot analysis in 4 and 6 weeks postoperatively. Western blot was performed according to the manufacturer's protocol. The primary antibodies including anti-RUNX2 (1:1,000), anti-ALP $(1 \mu \mathrm{g} / \mathrm{ml})$, antiVEGF $(5 \mu \mathrm{g} / \mathrm{ml})$, anti-TGF- $\beta 1$ (1:300), and $\beta$-actin (1:5,000) were obtained from Abcam (Cambridge, UK). The immunoreactive bands were visualized and finally quantified using Quantity One Software. And all values were normalized to the value of $\beta$-actin.

\section{Statistical analysis}

All data were reported as means with standard deviations, and analyzed by SPSS 13.0 software (SPSS Inc, Chicago, IL, USA). Differences were considered as significant at $P \leq 0.05$. For normally distributed data, Student t test or one-way analysis of variance was used to compare differences between 2 different groups or among more than 2 groups.

\section{Results}

\section{$\mathrm{X}$-ray measurement}

Immediately after surgery, as presented in Fig. 2A-C, segmental left radius shaft defects were fixed with PMMA and plates, PMMA only, or nothing, suggesting that these models were successful. Both plates and PMMA were radiopaque so that the bone defects and the implant materials were clearly visible. Compared with immediate and 4 weeks postoperative radiographs (Fig. 2A-F), there was no sign of plates 
loosening, or shift of PMMA. As shown in Fig. 2F, no radiopaque growth was observed in the control group 4 weeks postoperatively. And the reduction of both ends of fracture was in accordance with that immediately after operation indicated that there was no significant movement between the fracture ends.

\section{Gross histology}

Gross observation showed the translucent and elastic membrane was formed around PMMA (Fig. 1F). In the control group, there was no membrane formation but callus tissue surrounding with connective tissue. The induced membrane (Fixation group and Non-fixation group) and connective tissue (control group) was removed completely for further testing. As shown in Fig. 3, H\&E staining revealed an intensive fibrous, cell-rich, and vascularized tissue in PMMA groups (Fixation group and Non-fixation group), while just little vessel like structure was observed in control group. Compared with membrane in Non-fixation group, there were more micro-vessels in Fixation group. And with the extending of implantation time, the micro-vessels had a tendency to increase and to be more mature. The osteogenesis activity was assessed via Alizarin red staining for calcium deposition. The results showed that the reaction of calcifying nodules was positive in Fixation group and Non-fixation group (Fig. 4), indicating excellent osteogenic activity.

\section{Proliferation}

Content of cell proliferation in the induced membranes and in the connective tissue were measured using $\mathrm{IHC}$ analysis by detection the percentage of Ki67 positive cells. As shown in Fig. 5, the positive cells displayed brown yellow or brown particles, and mainly distributed in the periphery of membrane. The results indicated that Ki67 positive cells were relatively high in the induced membranes, but that was little in the connective tissue. And a slightly increased proliferative activity was observed in membranes of Fixation group in comparison to that of Non-fixation group. A qualitative trend toward increased percentage of Ki67 positive cells was observed in PMMA groups between 4 weeks and 6 weeks.

\section{Osteogenesis}

Content of osteogenesis in the induced membranes and in the connective tissue were measured using IHC analysis by detection COL I. As shown in Fig. 6, the positive cells displayed brown yellow or brown particles, and mainly scattered in the membrane. The qualitative IHC analysis shown that the expression of $\mathrm{COL} I$ increased evidently than that of the control group, and that there was no obvious difference in the percentage of COL I between the two PMMA groups in 4weeks and 6 weeks after operation.

As shown in Fig. 7, Western blot analysis revealed that the membrane extracted from Fixation group and Non-fixation group both exhibited higher protein expression of Runx2 and ALP compared to the control group, 4 and 6 weeks postoperatively. Additionally, in comparison to membrane in Non-fixation group, an increasing trend in the protein expression of Runx2 and ALP was observed in Fixation group, although the difference did not reach significance in the IM group on day 7 (Fig. 2B-D). Western blot analysis also 
revealed that both the IM and BMP-2 exert a significant effect on the protein expression of Runx2, Col 1, and OCN, particularly in the BMP-2 group (Fig. 2E and 2F). Western blot was almost consistent with the $\mathrm{IHC}$ results.

\section{Vascularization}

Content of vascularization in the induced membranes and in the connective tissue were measured using $\mathrm{IHC}$ analysis by detection the percentage of CD31 positive cells. As shown in Fig. 8, the positive cells displayed brown yellow particles, mainly scattered, and formed tube-like vascular structure in the membrane. The results indicated that CD31 positive cells were relatively high in the induced membranes, but that in the connective tissue was little. The comparison between Fixation group and Non-fixation group did not reveal any significant differences postoperatively. And with the extending of implantation time, the CD31 positive cells formed the more mature organized micro-vessels in 6 weeks after operation.

\section{Discussion}

There were many researches regarding that the stability of fracture broken end directly affects the healing of bone fracture(25-28). While during the first phase of Masquelet, the bone defects filling with PMMA spacer are fixed with internal plate, intramedullary nail, external fixator, casting, or nothing $(17,19-21)$. Each fixed mode could result in a different mechanical environment, subsequently have significant influence on bone repair. No study has been done yet to evaluate the effect of PMMA spacer stability on subsequently bone formation in the membrane cavity. This was the first study to investigate the influencing of topical mechanical stability of fracture broken end on the subsequently formation of Masquelet membrane. In order to explore the specific mechanisms, two fixation variables, rigid fixation offered by plates and relative fixation by suturing, were selected for further research.

The rabbit models of bone defects were commonly used in laboratory experiments, and stainless-steel internal plate was usually applied for the fixation of bone defect ends $(9,29,30)$. Through X-ray measurement postoperatively we confirmed that the Masquelet models with plate internal fixation was successfully made. The X-ray results suggested no significant movement between the fracture ends, even in the control group without PMMA and plate. Due to the integrity of ulna and interosseous membrane, there was no visible displacement of fracture segments(19). And due to the supporting of PMMA in defects, and suture tension of fascia, PMMA in Non-fixation group was in relatively stable pattern with little distortions(30). Still, the jiggle of fracture fragments was existed theoretically, which might have impacts on subsequently membrane formation.

Through gross histology we found that a translucent and membrane-like fibrotic capsule was formed around PMMA, and that connective tissue formed in the bone defects in the control group, which is consistent with the literature reported $(15,19)$. H\&E and Alizarin red staining results suggested that the intensive fibrous, cell-rich, calcium-positive, and vascularized membrane around PMMA would be more conducive to osteogenic activity. 
Content of proliferation was assessed by detection the percentage of Ki67 positive cells. Ki67 is a core protein seen in proliferating cells, which exhibits good morphological properties of cell proliferation(31). The proliferation was slightly higher in the induced membranes compared to the control groups, and a slightly increased proliferative activity was observed in membranes of Fixation group in comparison to that of Non-fixation group, suggesting that the mechanical stability offered by plates was beneficial to cell proliferation in the induced membrane. The results was corresponding to previous research that the cell proliferation was relatively active in the membrane $(15,19)$. Besides, our research found that a qualitative trend toward increased proliferation was observed in PMMA groups between 4 weeks and 6 weeks.

The Col I was selected for osteogenesis qualitatively. And the expression levels of ALP and RUNX2 were used for the quantitative analysis of osteogenic activity, of which ALP is an early sign of osteoblast differentiation and maturation, and RUNX2 plays a central role in coordinating multiple signals involved in osteoblast activity(32). By comparing results between PMMA groups and the control group, osteogenic activity of the induced membranes was obviously expressed during the whole observation period. Similar animal results were obtained from previous studies. It is well-known that the different mechanical stabilities around bone defects lead to different callus responses and osteoblast activities(33). And current clinical findings indicate that appropriate fixation of the bone defect is desirable in Masquelet technique, with the use of internal fixator, external fixator, or plaster cast $(1,3,7)$. Still, the exact mechanism remains unknown. In our research, an increasing trend in the protein expression of Runx2 and ALP was observed in Fixation group, in comparison to Non-fixation group. These results revealed that the rigid fixation provided by internal plate could further promote osteogenesis, which implying that the use of fixation in Masquelet technique has potential clinical benefits.

The detection of CD31 positive cells represents the formation of new vessels. And the vascularity of the membrane was quantitatively evaluated by detecting the level of VEGF and TGF- $\beta 1(16,34)$. Previous researches have reported that the tube-like vascular structure is formed in the induced membrane around PMMA, and the highly vascular membrane has many similarities to the periosteum $(13,35)$. The present study indicated that CD31 positive cells were relatively high in the induced membranes, and the higher level of CD31 expression signals greater capacity of vascularization. And the relative expression levels of VEGF and TGF- $\beta 1$ in membrane around PMMA were higher than those in connective tissue of the control group. Besides, significant differences were found between Fixation and Non-fixation group, suggesting that the rigid fixation provided by internal plate could further promote vascularization too.

Exploring the influencing of topical mechanical stability on subsequently membrane formation could help to further the clinical applications. Further experiments and clinical research to verify their effectiveness would help to further understand the exact generating and regulatory mechanism in Masquelet technique and help to improve clinical outcomes. And the next step in this research would be to further validate if rigid fixation benefits bone defect repairing in the second stage of Masquelet.

\section{Conclusion}


The results of the current study clearly indicate that rigid fixation provided by internal plate in Masquelet technique positively alters the quality of membrane formed surrounding PMMA, in terms of significantly osteogenic and angiogenic factors expression, and greater potency of bone formation in the second stage of Masquelet. Specific as follow: Membrane surrounding PMMA has some osteogenic and angiogenic potential in Fixation group and Non-fixation group; Compared with membrane in Non-fixation group and Control group, pathological examinations suggest that the relatively more vascularized and osteogenic membrane was formed in Fixation group, and HIC results suggest that the expression of Ki67, COL I, and CD31 positive cells increased evidently; Fixing PMMA spacer with internal plate causes a significant increase in osteogenic factors (RUNX2 and ALP) and angiogenic factors (VEGF and TGF- $\beta 1$ ) in 4 and 6 weeks postoperatively.

\section{List Of Abbreviations}

Polymethylmethacrylate, PMMA; Immunohistochemistry, IHC.

\section{Declarations}

\section{Ethics approval and consent to participate}

The medical ethics committee of Xiangya Hospital Central South University gave ethical approval of this research.

\section{Consent for publication}

All patients gave written informed consent to involvement in this study, including consent to use data from medical records and radiographs.

\section{Availability of data and material}

Data sharing is not applicable to this article as no datasets were generated or analysed during the current study.

\section{Competing interests}

No conflict of interest exits in the submission of this manuscript, and all authors are fully involved in the study and preparation of the manuscript.

\section{Funding}

None

Authors' contributions 
Experimental design: Zeng Min, Xie Jie, Zhu Yong. Performing rabbit experiments: Zeng Min, Xie Jie, Liu Donghao, Wang Haoyi. Drafting of the article: Zeng Min, Xie Jie, Hu Yihe. Histological detection: Zeng Min, Long Haitao. Radiography: Zhu Yong, Cheng Liang. Western blot analysis: Zeng Min, Xie Jie. All authors read and approved the final manuscript.

\section{Acknowledgements}

None

\section{Conflict of Interest}

The authors declare that they have no conflict of interest.

\section{Reference}

1. Giannoudis PV, Faour O, Goff T, Kanakaris N, Dimitriou R. Masquelet technique for the treatment of bone defects: tips-tricks and future directions. Injury. 2011;42(6):591-8.

2. Mauffrey C, Giannoudis PV, Conway JD, Hsu JR, Masquelet AC. Masquelet technique for the treatment of segmental bone loss have we made any progress? Injury. 2016;47(10):2051-2.

3. Morris R, Hossain M, Evans A, Pallister I. Induced membrane technique for treating tibial defects gives mixed results. Bone Joint J. 2017;99-B(5):680-5.

4. Masquelet AC. Muscle reconstruction in reconstructive surgery: soft tissue repair and long bone reconstruction. Langenbecks Arch Surg. 2003;388(5):344-6.

5. Masquelet AC, Fitoussi F, Begue T, Muller GP. [Reconstruction of the long bones by the induced membrane and spongy autograft]. Ann Chir Plast Esthet. 2000;45(3):346-53.

6. Roukoz S, El Khoury G, Saghbini E, Saliba I, Khazzaka A, Rizkallah M. Does the induced membrane have antibacterial properties? An experimental rat model of a chronic infected nonunion. Int Orthop. 2019.

7. Morelli I, Drago L, George DA, Gallazzi E, Scarponi S, Romano CL. Masquelet technique: myth or reality? A systematic review and meta-analysis. Injury. 2016;47 Suppl 6:S68-S76.

8. Ronga M, Cherubino M, Corona K, Fagetti A, Bertani B, Valdatta L, et al. Induced membrane technique for the treatment of severe acute tibial bone loss: preliminary experience at medium-term follow-up. Int Orthop. 2019;43(1):209-15.

9. Viateau V, Bensidhoum M, Guillemin G, Petite H, Hannouche D, Anagnostou F, et al. Use of the induced membrane technique for bone tissue engineering purposes: animal studies. Orthop Clin North Am. 2010;41(1):49-56; table of contents.

10. Gouron R, Petit L, Boudot C, Six I, Brazier M, Kamel S, et al. Osteoclasts and their precursors are present in the induced-membrane during bone reconstruction using the Masquelet technique. $J$ Tissue Eng Regen Med. 2017;11(2):382-9. 
11. Pelissier P, Masquelet AC, Bareille R, Pelissier SM, Amedee J. Induced membranes secrete growth factors including vascular and osteoinductive factors and could stimulate bone regeneration. $J$ Orthop Res. 2004;22(1):73-9.

12. Henrich D, Seebach C, Nau C, Basan S, Relja B, Wilhelm K, et al. Establishment and characterization of the Masquelet induced membrane technique in a rat femur critical-sized defect model. $\mathrm{J}$ Tissue Eng Regen Med. 2016;10(10):E382-E96.

13. McBride-Gagyi S, Toth Z, Kim D, Ip V, Evans E, Watson JT, et al. Altering spacer material affects bone regeneration in the Masquelet technique in a rat femoral defect. J Orthop Res. 2018.

14. Tarchala M, Engel V, Barralet J, Harvey EJ. A pilot study: Alternative biomaterials in critical sized bone defect treatment. Injury. 2018;49(3):523-31.

15. Nau C, Seebach C, Trumm A, Schaible A, Kontradowitz K, Meier S, et al. Alteration of Masquelet's induced membrane characteristics by different kinds of antibiotic enriched bone cement in a critical size defect model in the rat's femur. Injury. 2016;47(2):325-34.

16. Ma YF, Jiang N, Zhang X, Qin CH, Wang L, Hu YJ, et al. Calcium sulfate induced versus PMMAinduced membrane in a critical-sized femoral defect in a rat model. Sci Rep. 2018;8(1):637.

17. Gaio N, Martino A, Toth Z, Watson JT, Nicolaou D, McBride-Gagyi S. Masquelet technique: The effect of altering implant material and topography on membrane matrix composition, mechanical and barrier properties in a rat defect model. J Biomech. 2018;72:53-62.

18. Sasaki G, Watanabe Y, Miyamoto W, Yasui Y, Morimoto S, Kawano H. Induced membrane technique using beta-tricalcium phosphate for reconstruction of femoral and tibial segmental bone loss due to infection: technical tips and preliminary clinical results. Int Orthop. 2018;42(1):17-24.

19. Yilmaz O, Ozmeric A, Alemdaroglu KB, Celepli P, Hucumenoglu S, Sahin O. Effects of concentrated growth factors (CGF) on the quality of the induced membrane in Masquelet's technique - An experimental study in rabbits. Injury. 2018;49(8):1497-503.

20. Toth Z, Roi M, Evans E, Watson JT, Nicolaou D, McBride-Gagyi S. Masquelet Technique: Effects of Spacer Material and Micro-topography on Factor Expression and Bone Regeneration. Ann Biomed Eng. 2019;47(1):174-89.

21. Luangphakdy V, Elizabeth Pluhar G, Piuzzi NS, D'Alleyrand JC, Carlson CS, Bechtold JE, et al. The Effect of Surgical Technique and Spacer Texture on Bone Regeneration: A Caprine Study Using the Masquelet Technique. Clin Orthop Relat Res. 2017;475(10):2575-85.

22. Taylor BC, French BG, Fowler TT, Russell J, Poka A. Induced membrane technique for reconstruction to manage bone loss. J Am Acad Orthop Surg. 2012;20(3):142-50.

23. Bosemark P, Perdikouri C, Pelkonen $M$, Isaksson $H$, Tagil $M$. The masquelet induced membrane technique with BMP and a synthetic scaffold can heal a rat femoral critical size defect. J Orthop Res. 2015;33(4):488-95.

24. Siboni R, Joseph E, Blasco L, Barbe C, Bajolet O, Diallo S, et al. Management of septic non-union of the tibia by the induced membrane technique. What factors could improve results? Orthop Traumatol Surg Res. 2018;104(6):911-5. 
25. Mark H, Nilsson A, Nannmark U, Rydevik B. Effects of fracture fixation stability on ossification in healing fractures. Clin Orthop Relat Res. 2004(419):245-50.

26. McKibbin B. The biology of fracture healing in long bones. J Bone Joint Surg Br. 1978;60-B(2):15062.

27. Santolini E, West R, Giannoudis PV. Risk factors for long bone fracture non-union: a stratification approach based on the level of the existing scientific evidence. Injury. 2015;46 Suppl 8:S8-S19.

28. Yu YY, Bahney C, Hu D, Marcucio RS, Miclau T, 3rd. Creating rigidly stabilized fractures for assessing intramembranous ossification, distraction osteogenesis, or healing of critical sized defects. J Vis Exp. 2012(62).

29. Meinig RP, Rahn B, Perren SM, Gogolewski S. Bone regeneration with resorbable polymeric membranes: treatment of diaphyseal bone defects in the rabbit radius with poly(L-lactide) membrane. A pilot study. J Orthop Trauma. 1996;10(3):178-90.

30. Meng ZL, Wu ZQ, Shen BX, Li HB, Bian YY, Zeng L, et al. Reconstruction of large segmental bone defects in rabbit using the Masquelet technique with alpha-calcium sulfate hemihydrate. $J$ Orthop Surg Res. 2019;14(1):192.

31. Scholzen T, Gerdes J. The Ki-67 protein: from the known and the unknown. J Cell Physiol. 2000;182(3):311-22.

32. Franceschi RT, Xiao G. Regulation of the osteoblast-specific transcription factor, Runx2: responsiveness to multiple signal transduction pathways. J Cell Biochem. 2003;88(3):446-54.

33. Probst A, Jansen $H$, Ladas A, Spiegel HU. Callus formation and fixation rigidity: a fracture model in rats. J Orthop Res. 1999;17(2):256-60.

34. Ferrara N, Gerber HP, LeCouter J. The biology of VEGF and its receptors. Nat Med. 2003;9(6):669-76.

35. Cuthbert RJ, Churchman SM, Tan HB, McGonagle D, Jones E, Giannoudis PV. Induced periosteum a complex cellular scaffold for the treatment of large bone defects. Bone. 2013;57(2):484-92.

\section{Figures}




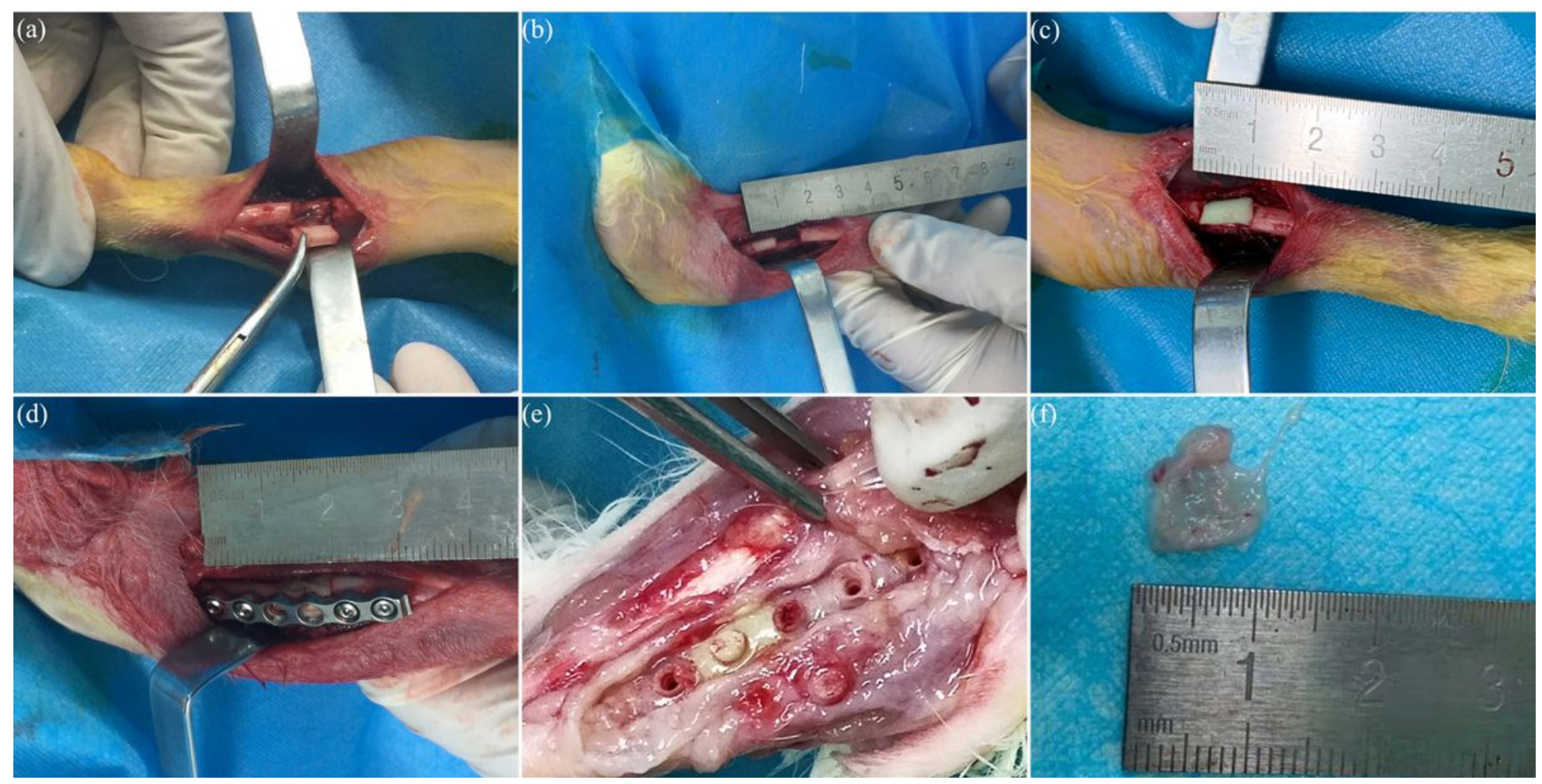

\section{Figure 1}

Application of the Masquelet technique. (a, b) Segmental left radius shaft defect (length, $9 \mathrm{~mm}$ ) was created. (c) Bone defect was filled with PMMA spacer $(3 \mathrm{~mm} \emptyset \times 9 \mathrm{~mm})$. (d) PMMA was fixed with stainless steel plates. (e, f) Masquelet membrane was formed surrounding the cement, 4 or 6 weeks postoperatively.

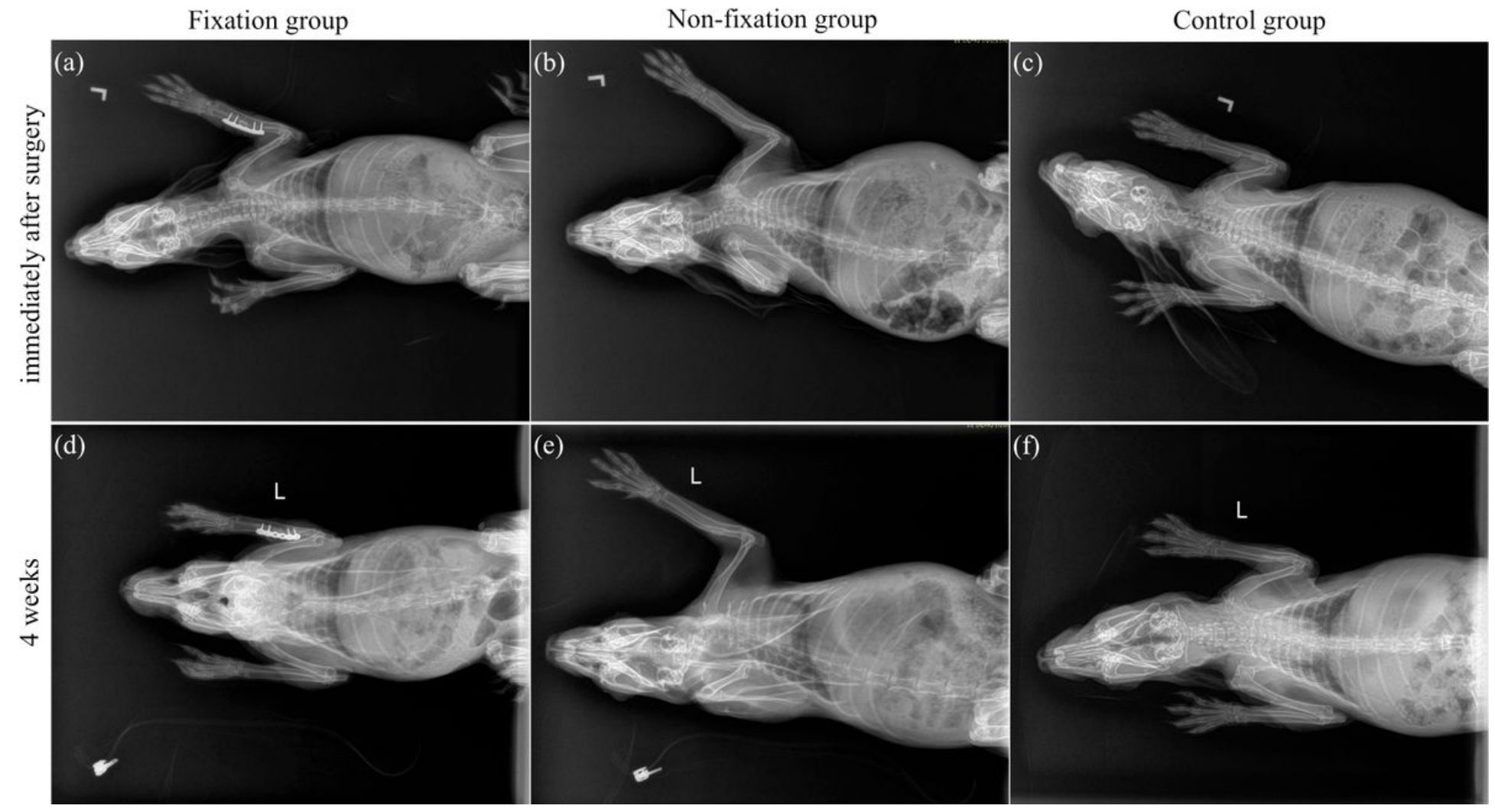




\section{Figure 2}

Postoperative X-ray images. (a-c) X-ray images shown that segmental left radius shaft defects were respectively fixed with PMMA and plates, PMMA only, or nothing, immediately after surgery. (d-f) There was no sign of plates loosening, or shift of PMMA, or immigration of fracture ends, 4 weeks postoperatively.

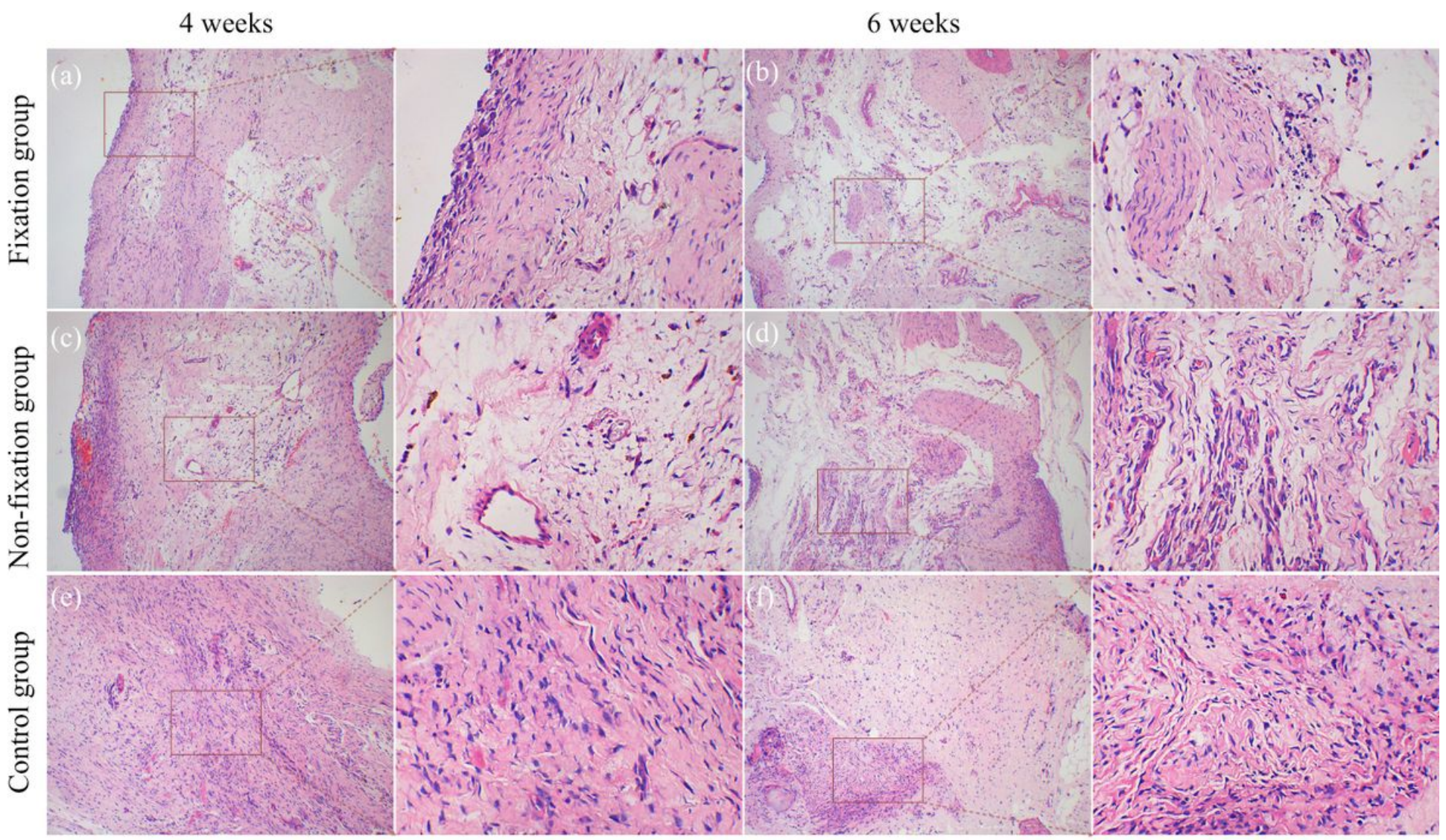

\section{Figure 3}

Representative H\&E staining of Masquelet membrane (magnification $* 100$ ). H\&E staining shown that the intensive fibrous, cell-rich, and vascularized tissue were formed in Fixation group (a) and Non-fixation group (c), while just little vessel like structure was observed in control group (e), in 4 weeks postoperatively. The micro-vessels had a tendency to increase and to be more mature in Fixation group (b) and Non-fixation group (d), while still little in control group (f), in 6 weeks postoperatively. 


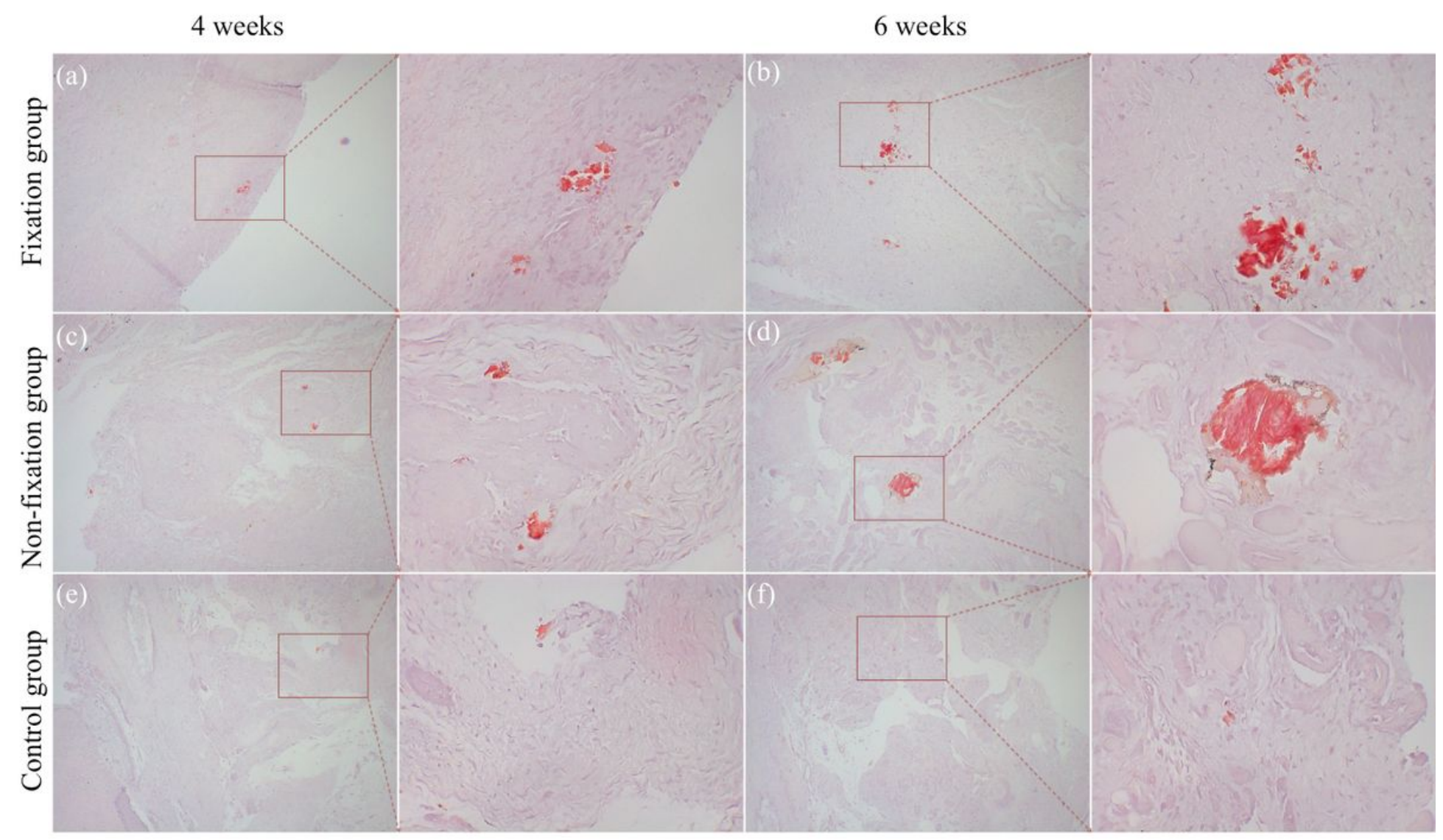

\section{Figure 4}

Representative Alizarin red staining of Masquelet membrane (magnification $* 100$ ). Calcium deposition was observed in Fixation group $(a, b)$ and Non-fixation group (c, d), while just little in control group (e, f), 4 and 6 weeks post-operation, respectively. 


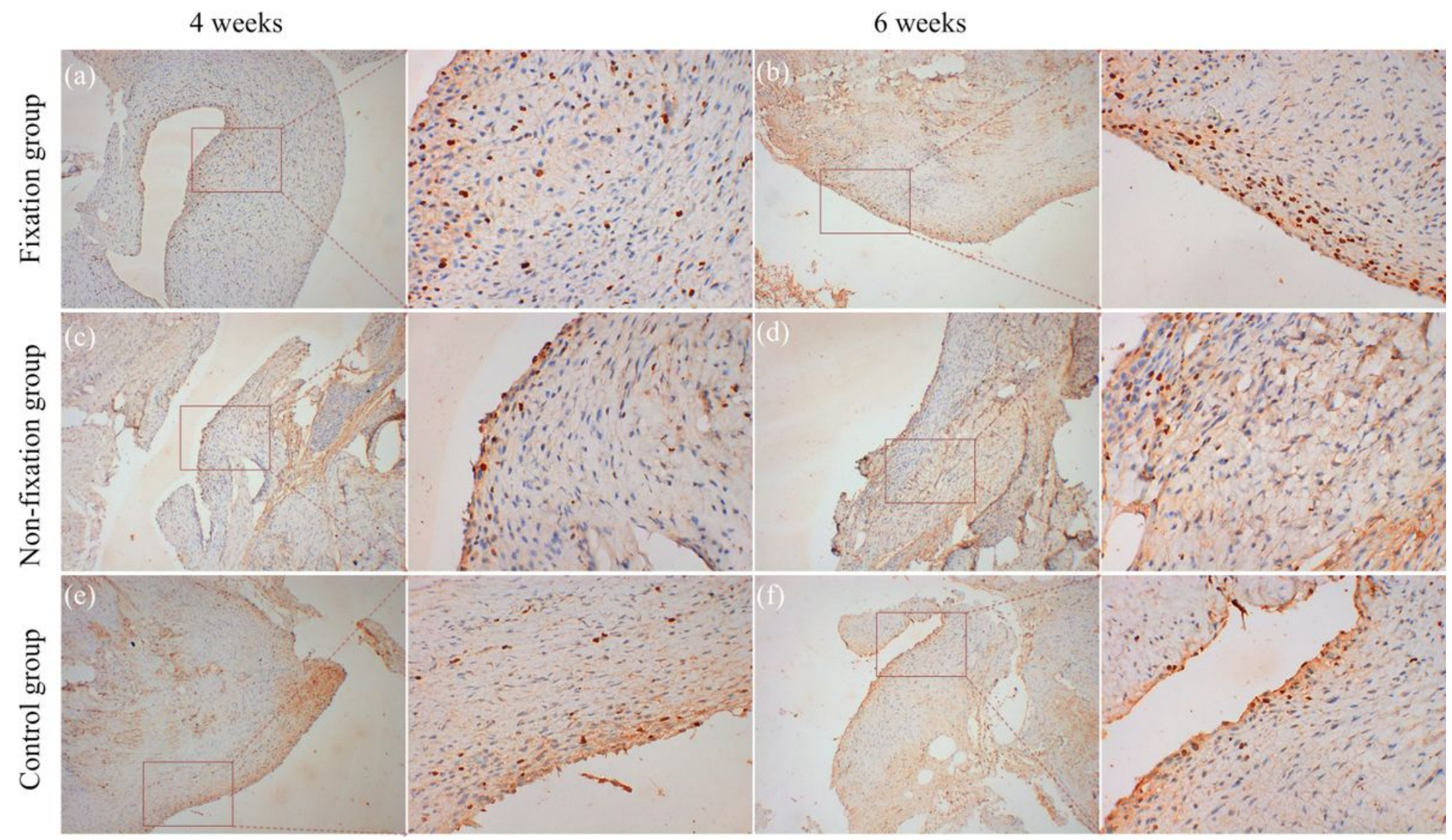

Figure 5

Representative IHC analysis of Ki67 (magnification * 100). (a-f) The Ki67 positive cells displayed brown yellow or brown particles, and mainly distributed in the periphery of membrane in 4 or 6 weeks postoperatively. 


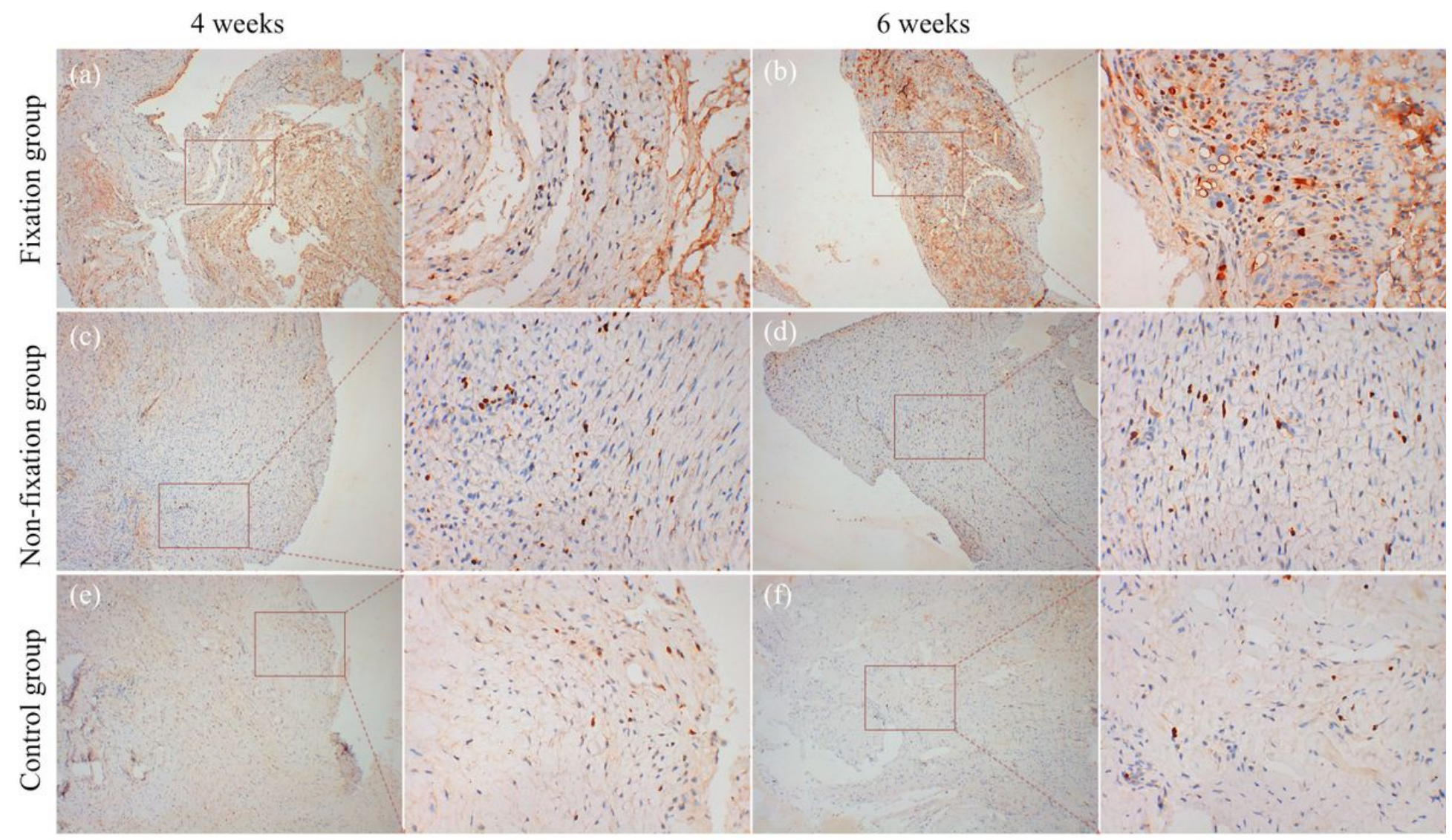

\section{Figure 6}

Representative IHC analysis of COL I (magnification $* 100$ ). (a-f) The COL I positive cells displayed brown yellow or brown particles, and mainly scattered in the membrane in 4 or 6 weeks postoperatively. 


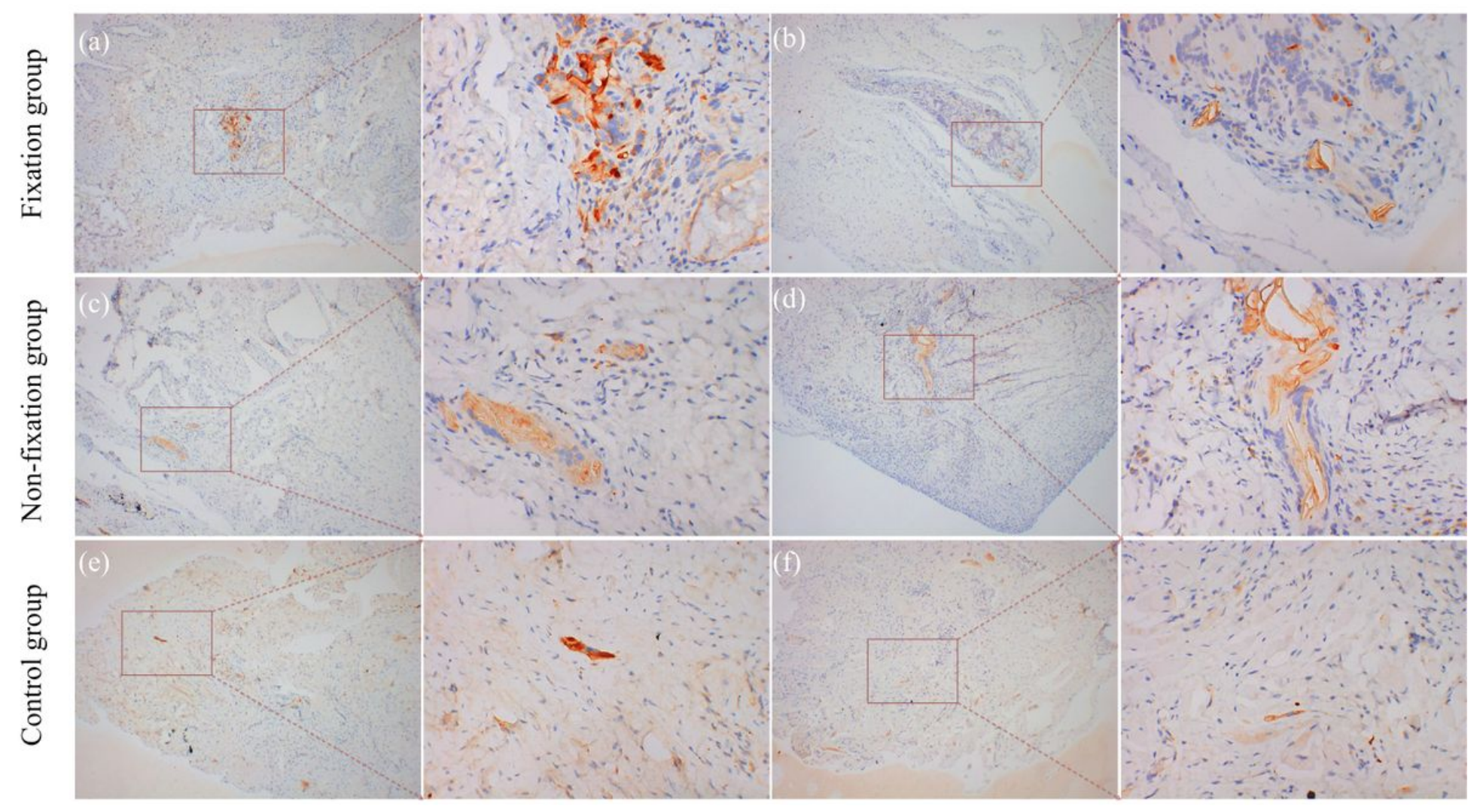

\section{Figure 7}

Representative IHC analysis of CD31 (magnification $* 100$ ). (a-f) The COL I positive cells displayed brown yellow particles, mainly scattered, and formed tube-like vascular structure in the membrane in 4 or 6 weeks postoperatively. 
(a)

RUNX2

$57 \mathrm{KD}$

ALP

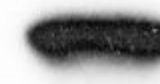

VEGFA

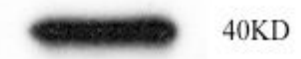

TGF- $\beta 1$

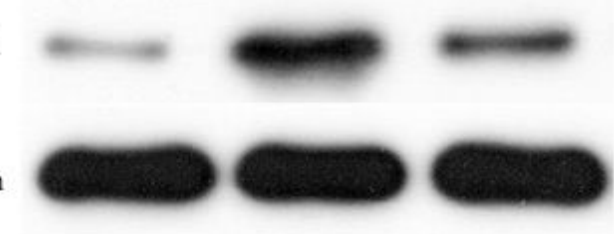

$12 \mathrm{KD}$

$\beta$-actin
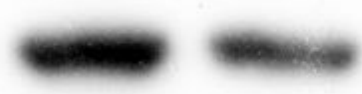

23KD

$42 \mathrm{KD}$

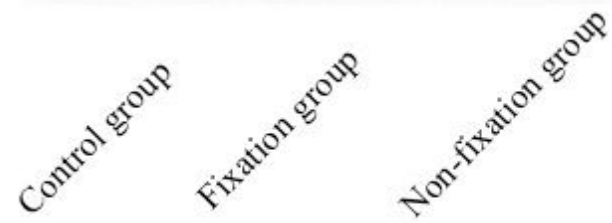

(c)

RUNX2

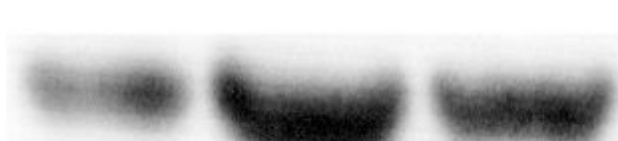

$57 \mathrm{KD}$

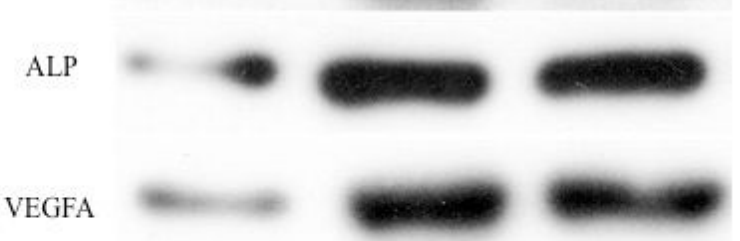

$40 \mathrm{KD}$

$23 \mathrm{KD}$

TGF- $\beta 1$

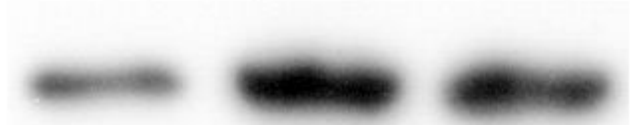

$\beta$-actin

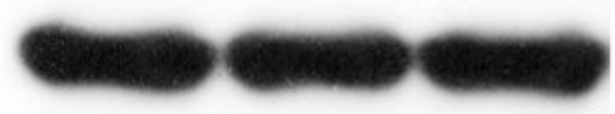

$12 \mathrm{KD}$
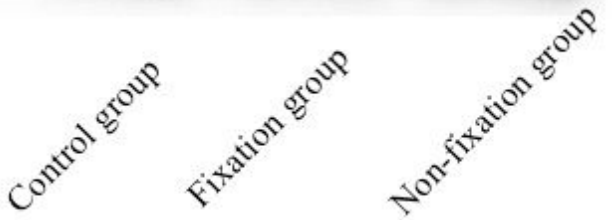

(b)

Control group

Fixation group

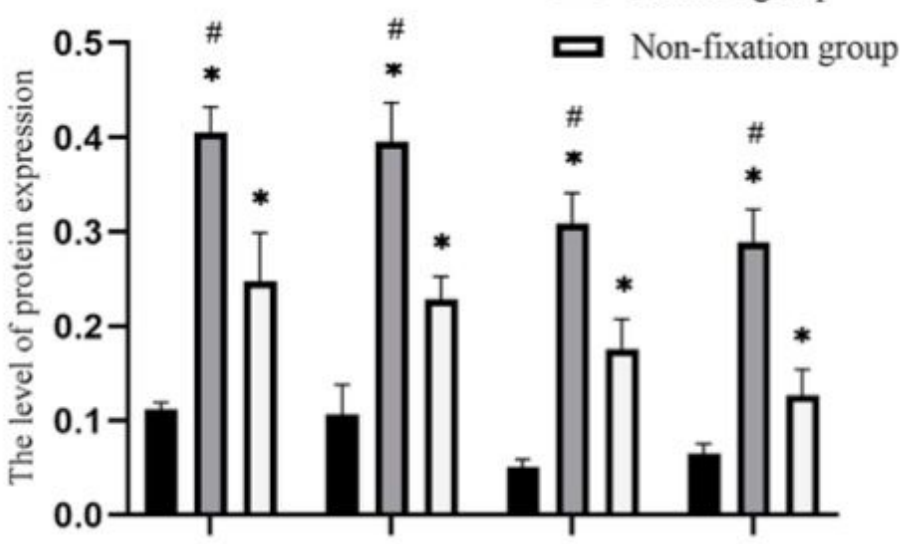

(d)
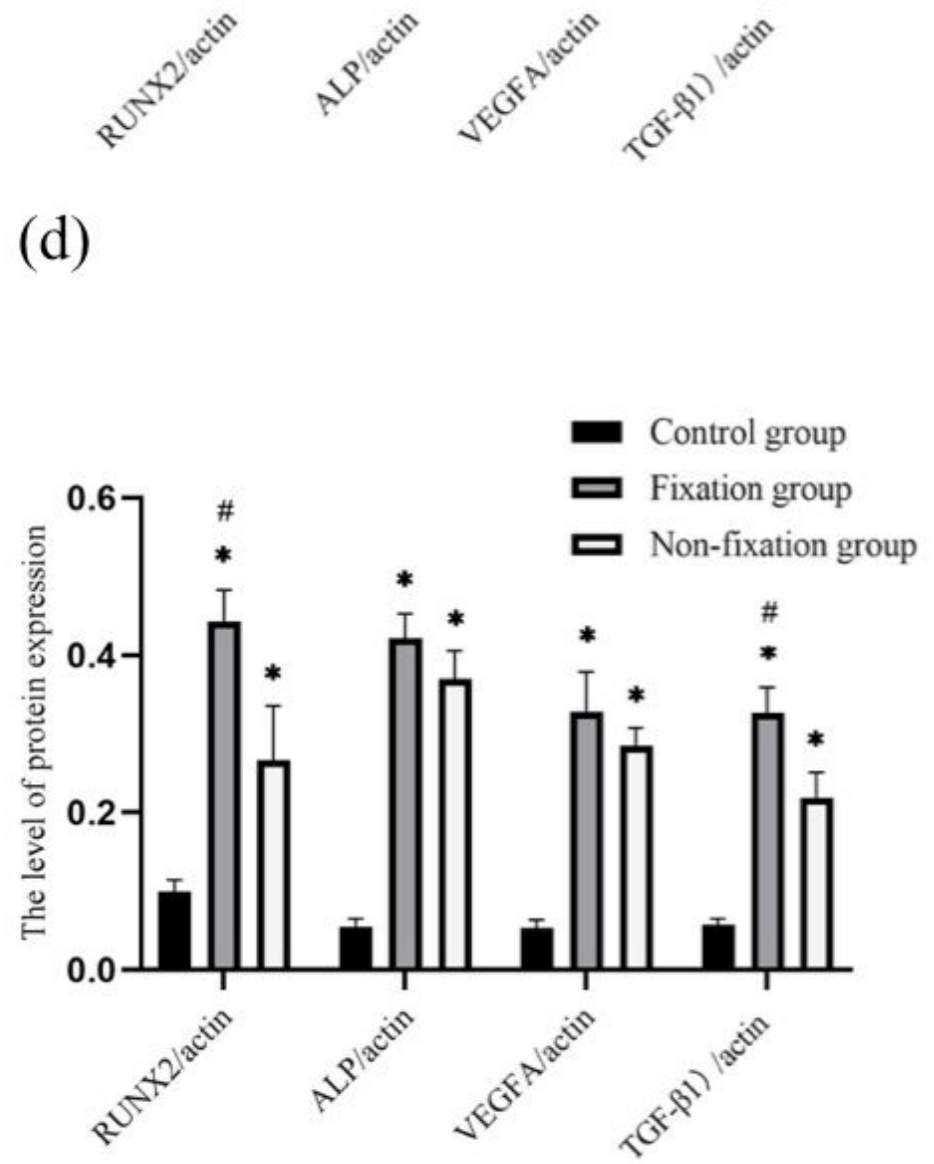

Figure 8

Western-Blotting analysis of osteogenic proteins (RUNX2 and ALP) and angiogenic proteins (VEGFA and TGF- $\beta 1)$. (a, b) 4 weeks postoperatively. (c, d) 6 weeks postoperatively. Significant differences between Fixation group and Non-fixation group groups are indicated as \# $(P<0.05)$. And significant differences between PMMA groups (including Fixation group and Non-fixation group) and Non-fixation group groups are indicated as * $(P<0.05)$. For each group, $n=6$. 